\title{
Emissions Impacts of Using Energy Storage for Power System Reserves
}

\author{
Yashen Lin $^{\mathrm{a}, \mathrm{b}, *}$, Jeremiah X. Johnson ${ }^{\mathrm{b}}$, Johanna L. Mathieu ${ }^{\mathrm{a}}$ \\ ${ }^{a}$ Department of Electrical Engineering and Computer Science, University of Michigan, 1301 Beal Ave, Ann Arbor, \\ MI 48109, USA \\ ${ }^{b}$ Center for Sustainable Systems, School of Natural Resources 85 Environment, University of Michigan, 440 \\ Church Street Ann Arbor, MI 48109, USA
}

\begin{abstract}
Energy storage devices, such as batteries and flywheels, are promising options for providing operating reserves due to their fast response and low emissions during operation. However, because of the complex nature of power systems, adding energy storage-based reserves to the power system may not necessarily benefit the environment. In this paper, we analyze these impacts in a test system and identify important drivers that affect the environmental outcomes. Dispatch results are obtained by solving an optimal power flow (OPF) problem and are used to determine emissions. We find that the impacts of adding energy storage are highly case-dependent. In systems with high renewable penetration levels and significant renewable curtailment, adding energy storage reduces emissions; in other systems, the impacts on emissions could be positive, neutral, or negative. The analyses presented in this paper show that policies to procure energy storage as a means to reduce emissions may actually lead to increased system-wide emissions if current dispatch algorithms are used. We also explore the impacts of modifying the dispatch algorithm to ensure system emissions with energy storage are no worse than system emissions without energy storage.

Keywords: Energy storage, operating reserves, emissions, optimal power flow
\end{abstract}

\footnotetext{
*Corresponding author. Tel.: +1-734-615-9789.

Email addresses: yashenl@umich.edu (Yashen Lin), jxjohns@umich.edu (Jeremiah X. Johnson), jlmath@umich.edu (Johanna L. Mathieu)
} 


\section{Introduction}

As more renewable energy sources are integrated into the power system, additional operating reserves are required to ensure the functionality and reliability of the system [1-4]. Conventional generators provide reserves by holding back some capacity so that they can ramp up or down to balance supply and demand. However, this can result in less efficient generator operation and increased air pollution $[5,6]$. Energy storage devices are promising alternatives to generators for providing reserves because, in general, these resources are more responsive than conventional power plants, output no emissions during operation, and their costs are increasingly competitive $[7,8]$.

Recent regulatory developments are creating more favorable conditions for large-scale deployment of grid-connected energy storage. For example, the Federal Energy Regulatory Commission (FERC) Order No. 755 [9] requires Independent System Operators (ISOs) to take performance, i.e., both response speed and accuracy, into account when compensating ancillary services. Though this is a policy that is equally applied to all service providers, it benefits energy storage more than traditional generators because energy storage usually has shorter response times and better accuracy. FERC Order No. 784 [10] requires utilities to better account for transactions related to energy storage resources. At the state level, California is leading the way in energy storage policy by mandating procurement requirement of 1,325 MW for energy storage by 2020 from its three investor-owned utilities [11].

With low or no direct emissions during operation, having energy storage-based reserves in power systems is often assumed to reduce pollutant emissions to the environment [12, 13]. Some policy makers also consider emissions reduction as a motivation for encouraging energy storage installation; see, for example, the California's energy storage procurement mandate [11]. However, due to the complex nature of power systems and their operational strategies, the validity of this assumption is not clear. The objective of this paper is to investigate the range of emissions impacts resulting from use of energy storage-based reserves in the current power system. We find that energy storage-based reserves may lead to negative impacts under certain dispatch algorithms, system configurations, generation mixes, and reserve requirements.

In a power system, generation and reserve dispatch schedules are determined by solving the 
unit commitment problem and the economic dispatch problem [14]. The unit commitment problem determines which units will be committed during which hours, but does not specify their production levels. The economic dispatch problem, which is solved after the unit commitment problem, is usually posed as an optimal power flow (OPF) problem [15] and determines the most cost-efficient production levels and reserve capacities across the committed units. In current U.S. electricity markets, only the energy and reserve costs of the system are minimized, while the emissions impacts are not explicitly considered within these problems (though some emissions are indirectly limited/priced, for example, $\mathrm{SO}_{\mathrm{x}}$ is limited through a cap and trade program and $\mathrm{CO}_{2}$ is priced through voluntary carbon markets). A number of dispatch algorithms that consider environmental impacts have been proposed; for example, including emissions costs [16-18] or constraints [19]. However, these algorithms have not yet been adopted. As a result, although adding energy storage to the system could reduce the cost of the system, the environmental outcome may not necessarily improve.

In this paper, we focus on the economic dispatch problem and adopt a single-period deterministic OPF formulation. This formulation models the current practice, which suits our objective of investigating the emissions impacts of adding energy storage-based reserves to the current power system. The state of charge (SOC) of the energy storage affects its ability to continuously provide reserves. ISOs have proposed to use the real time energy market to manage the SOC of storage devices, so that they can bid their full capacity without considering their current SOC [20, 21]. This decouples the time periods of the OPF problem and justifies the use of a single-period formulation. Stochastic formulations have been proposed [22, 23]; however, they are not yet adopted in practice.

The OPF problem is solved to obtain the dispatch result, which is used for evaluating the $\mathrm{CO}_{2}$ emissions. We first analyze a standard IEEE 9-bus test system [24], which is modified to include a renewable energy source, energy storage, and congestion, and is simple enough for analysis. We then test a more realistic IEEE 30-bus system [24, 25]. Though the test systems do not capture all the complexities in a real power system, the similar trends between the 30-bus system and 9-bus system indicate that the overarching trends we find are generalizable to real systems.

Many researchers and practitioners have studied energy storage in power systems. However, 
the emissions impacts of energy storage-based reserves are usually studied for a particular power system or under a newly-proposed dispatch algorithm. How the impacts vary under different grid configurations given current dispatch practices have not been thoroughly investigated. In this paper, we analyze the emissions impacts of energy storage-based reserves under different grid configurations and identify important factors that affect these impacts. A few examples of dispatch strategies for power systems with energy storage include [26-32]. The economic potential of energy storage is studied in $[8,33,34]$. Researchers have also included energy storage-based reserves in largescale simulations; see, for example, [5]. In [12, 13], the authors discover that utilizing electric vehicle batteries to provide ancillary service could reduce emissions in simulations of a model of Electricity Reliability Council of Texas (ERCOT) electric power system. In [30], a hierarchical dispatch algorithm is proposed to include responsive loads as energy storage for spinning reserves; reduction in operating costs and emissions are observed in tests on a modified IEEE 14-bus system. Although in $[12,13,30]$, positive environmental impacts are reported in their particular test systems ([30] also includes carbon tax), our paper finds that emissions results are a function of the grid configuration and could decrease or increase with energy storage.

Our contributions are three-fold. First, we develop methods to evaluate the emissions impacts of adding energy storage-based reserves to a physically constrained power system. Second, we find that the emissions impacts of adding energy storage-based reserves are case-dependent and may not always be positive, depending on the system configuration. Third, our results inform energy policy. Energy storage policies are still in their infancy, but as interest in these technologies grows, one may expect an increase in such policies. Our results will help policy makers understand the potential environmental outcomes resulting from use of energy storage-based reserves, allowing for better design of incentives and dispatch algorithms that achieve the over-arching environmental and economic goals.

The rest of the paper is organized as follows: Section 2 describes the problem formulation, assumptions, and simulation setup; Section 3 presents the simulation results and analyses; Section 4 concludes the paper. 


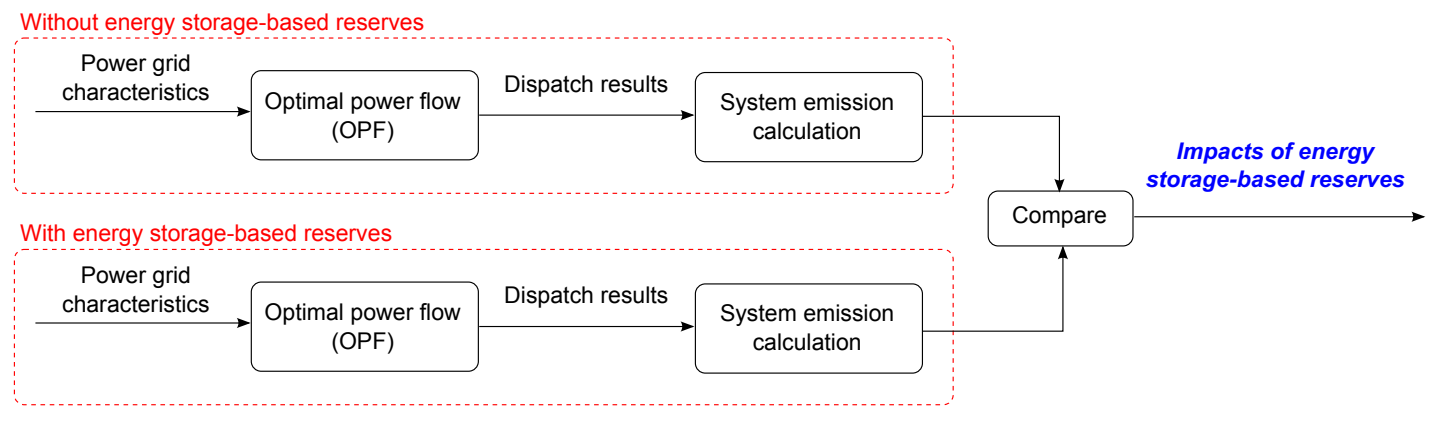

Figure 1: Flowchart for evaluating emissions impacts of adding energy storage-based reserves.

\section{Methods}

\subsection{Problem formulation}

In the operation of power systems, the majority of pollutant emissions are from fuel combustion $[35,36]$. The amount of fuel that is consumed is directly determined by generator operation. In U.S. electricity markets, the generation and reserve dispatch schedules are determined by solving the OPF problem. Information required for this dispatch decision, such as generator bids, generator limits, and grid topology, is collected by the system operator, who schedules generation and reserve capacity for each unit while minimizing the system cost. Once the dispatch results are determined, we can evaluate the system emissions. To study the impacts of adding energy storage-based reserves to the system, we first solve the OPF problem without energy storage-based reserves, and then solve it again with energy storage-based reserves. The impact is the difference between the two. A flowchart of our method is shown in Figure 1.

\subsection{Assumptions}

In this paper, we make the following general assumptions (a few OPF-related assumptions are introduced in Section 2.3):

1. We analyze $\mathrm{CO}_{2}$ emissions only; other pollutants, such as such as $\mathrm{NO}_{\mathrm{x}}$ and $\mathrm{SO}_{\mathrm{x}}$, could be analyzed with a similar method. 
2. The reference signal for reserves is zero-mean and the SOC of the energy storage devices are managed in the real time energy market, which decouples the dispatch periods, enabling us to use a single period OPF. In practice, the reference signal for energy storage could be biased, which could result in the energy storage devices becoming completely full or empty and being unable to provide reserves. The ISOs are developing strategies to deal with this. For example, the California ISO has implemented Regulation Energy Management (REM), a functionality for Non-Generator Resources (NGRs) which uses the real time energy market to help NGRs maintain their desired state of charge [20].

3. Reserves are symmetric, meaning a unit has to provide the same amount of up and down reserve; this is a normal practice in several ISOs, such as the PJM Interconnections (PJM) [37];

\subsection{Optimal power flow}

The OPF problem is an optimization problem whose objective is to minimize power system operating cost while satisfying system constraints. The decision variables usually include the generator power injections at each bus and reserve capacities provided by each unit. In U.S. electricity markets, the cost function usually includes generation and reserve costs. The power flow equations are nonlinear; however, here, for simplicity, we use the linearized power flow equations [15], also 
known as the DC power flow equations. The OPF problem is:

$$
\begin{array}{ll}
\underset{X}{\operatorname{minimize}} & f^{g}\left(P^{g}\right)+f^{r}\left(R^{g}\right)+f^{E S}\left(R^{E S}\right) \\
\text { subject to } & P_{f}=B_{f} \Theta \\
& P_{b u s}=B_{b u s} \Theta \\
& P_{b u s}=C^{g} P^{g}-C^{d} P^{d}-C^{E S} P^{d, E S} \\
& \mathbf{1}^{T} R^{g}+\mathbf{1}^{T} R^{E S} \geq r_{\text {req }} \\
& P^{g}-R^{g} \succeq P_{\min }^{g} \\
& P^{g}+R^{g} \preceq P_{\max }^{g} \\
& R_{\text {min }}^{E S} \preceq R^{E S} \preceq R_{\max }^{E S} \\
& P_{f, \min } \preceq P_{f} \preceq P_{f, \max }
\end{array}
$$

where $\succeq$ and $\preceq$ denote element-wise inequalities and $\mathbf{1}^{T}$ is a vector of ones of appropriate dimension. The decision variable $X=\left[P^{g}, \Theta, R^{g}, R^{E S}\right]$ includes the power generation of each unit $P^{g} \in \mathbb{R}^{n_{g e n}}$, the voltage angle at each bus $\Theta \in \mathbb{R}^{n_{\text {bus }}}$, reserve capacity provided by each generator $R^{g} \in \mathbb{R}^{n_{\text {gen }}}$, and reserve capacity provided by each energy storage device $R^{E S} \in \mathbb{R}^{n_{E S}}$. The cost function is the sum of the total generation cost $f^{g}$ and the reserve costs $f^{r}$ and $f^{E S}$. The variable $P_{f} \in \mathbb{R}^{n_{\text {line }}}$ is the power flow through each transmission line, $P_{b u s} \in \mathbb{R}^{n_{b u s}}$ is the power injection into each bus. Matrices $B_{f}$ and $B_{\text {bus }}$ include the susceptances of the transmission lines. Parameter $P^{d} \in \mathbb{R}^{n_{\text {bus }}}$ is the load at each bus and $P^{d, E S} \in \mathbb{R}^{n_{\text {bus }}}$ is the load caused by energy storage losses at each bus. $C^{g}, C^{d}, C^{E S}$ are grid topology matrices mapping generators and energy storage to buses (the entry $C_{i, j}$ is one if unit $j$ is connected to bus $i$ and zero otherwise). Parameter $r_{r e q}$ is the reserve capacity required by the power system. Constraint (2) is the DC power flow equation, (3) computes the net power injections from the voltage angles, and (4) is the power balance equation. These three constraints describe how power is delivered from generators to loads through the transmission network. Constraint (5) is the reserve requirement for the system; (6) and (7) are the generator limits; (8) is the reserve limit for the energy storage devices; and (9) is the transmission line limit. 
Due to conversion losses, not all of the energy injected into an energy storage device can be retrieved. Thus, although the reference signal for reserves is assumed to be zero-mean, each storage device looks like a load. Let $W^{E S}$ be the energy injected into the energy storage during a time period of $\Delta t$. Also, through the paper, we use letters with subscript $i$ to denote scalar quantities corresponding to the $i^{t h}$ unit. Then, $W_{i}^{E S}$ can be expressed as:

$$
W_{i}^{E S}=k^{E S} R_{i}^{E S}
$$

where $k^{E S}$ is the energy injection per unit of reserve procurement during $\Delta t$, which is determined by the reference signal. Let the round trip efficiency $\beta$ be the ratio of energy retrieved from the energy storage device to energy injected into the energy storage device. Then, the loss introduced by providing reserves is:

$$
L_{i}\left(R_{i}^{E S}\right)=\left(1-\beta_{i}\right) W_{i}^{E S}=\left(1-\beta_{i}\right) k^{E S} R_{i}^{E S}
$$

In the OPF problem, we model this loss as an additional load $P_{i}^{d, E S}$ at each energy storage bus. The magnitude of $P_{i}^{d, E S}$ is $L_{i} / \Delta t$. Note that this model is a generic model for energy storage devices and is not technology-specific.

In this formulation, we assume the reserve actions do not violate the transmission line constraints. The reasoning behind this assumption is that line constraints are thermal constraints and are usually conservative [38]. Thus, violations caused by reserve actions for short periods of time are unlikely to cause transmission line failures. However, this assumption may not hold when the reference signal for reserves is slowly varying, in which case the line flow could be over the limit for a long period of time, damaging the transmission line. The relaxation of this assumption is discussed in Section 3.4, where we study the case in which reserve actions are included in the transmission line constraints. 


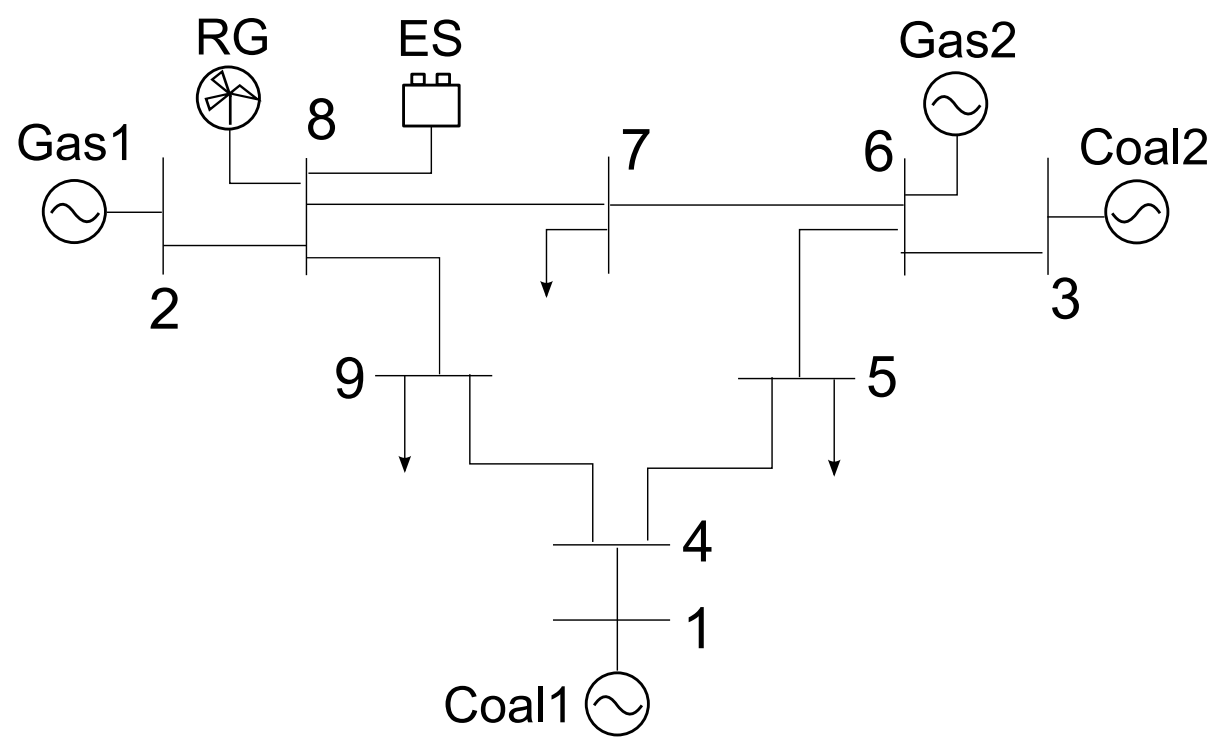

Figure 2: The IEEE 9-bus test system [24] modified to include a renewable generator and an energy storage device at bus 8 and an additional traditional generator at bus 6 . Each numbered segment is a bus and lines between buses are transmission lines. Arrows are loads. We assume each traditional generator is either a gas or coal power plant. RG refers to renewable generation; ES refers to energy storage.

\subsection{Test system}

In this paper, we analyze a simple 9-bus test system and a more realistic 30-bus system. The IEEE 9-bus system [24] is modified to include traditional generators with different types and sizes, a renewable energy source, and energy storage devices. The modified test system is shown in Figure 2. We added one traditional generator at bus 6 and one renewable energy source at bus 8 . The four traditional generators represent two coal units and two gas turbine units of different sizes. The generator limits are shown in Table 1. For demonstration, we choose one smaller and one larger coal unit, as well as one smaller and one larger gas turbine unit. The minimum operating point is assumed to be a portion of their maximum power outputs ( $40 \%$ for coal units, $50 \%$ for gas turbine units). We assume there is only one energy storage device, which is connected to bus 8 .

Fuel characteristics are needed to calculate generator costs and emissions. To obtain the fuel characteristics of the generators, the heat input-output data for generator Hunters Point 3 (a coal 
Table 1: Generator limits in the modified IEEE 9-bus test system.

\begin{tabular}{lccccc}
\hline & Coal 1 & Gas 1 & Coal 2 & Gas 2 & Renewable \\
\hline Minimum (MW) & 60 & 70 & 120 & 150 & 0 \\
Maximum (MW) & 150 & 150 & 300 & 300 & $200^{*}$ \\
\hline
\end{tabular}

* forecasted value.

unit) and Moss Landing 7 (a gas turbine unit) are collected from [39]. These data describe the fuel consumed by the unit at different levels of power output. For each of the two units, we fit the data to a second-order polynomial:

$$
F_{i}\left(P_{i}^{g}\right)=a_{i} P_{i}^{g 2}+b_{i} P_{i}^{g}+c_{i}
$$

where $F_{i}\left(P_{i}^{g}\right)$ is the fuel consumed by the $i^{t h}$ unit (in MMBtu/h) and $a_{i}, b_{i}$, and $c_{i}$ are calibrated parameters. If we divide $F_{i}\left(P_{i}^{g}\right)$ by $P_{i}^{g}$, we get the average heat rate of the unit, i.e.,

$$
H_{i}\left(P_{i}^{g}\right)=\frac{F_{i}\left(P_{i}^{g}\right)}{P_{i}^{g}}=a_{i} P_{i}^{g}+b_{i}+\frac{c_{i}}{P_{i}^{g}}
$$

Let $x=P_{i}^{g} / P_{\max , i}^{g}$ be the partial load ratio, we have:

$$
H_{i}(x)=a_{i} P_{\max , i}^{g} x+b_{i}+\frac{c_{i}}{P_{\max , i}^{g} x}
$$

The $x-H$ curve describes how partial load operation affects the unit's efficiency. The parameters for the generators in our test system are obtained by:

$$
\begin{aligned}
a_{i}^{F} & =a_{i} P_{\text {max }, i}^{g} \\
b_{i}^{F} & =b_{i} \\
c_{i}^{F} & =\frac{c_{i}}{P_{\text {max }, i}^{g}}
\end{aligned}
$$

With the scaled parameters $a_{i}^{F}, b_{i}^{F}$, and $c_{i}^{F}$, the $x-H$ curves of our coal units are set equivalent to that of the Hunters unit, and the $x-H$ curves of our gas turbine units are set equivalent to the 
Moss unit. Ignoring other operating costs, the generation cost is:

$$
f_{i}^{g}\left(P_{i}^{g}\right)=k_{c, i} F_{i}\left(P_{i}^{g}\right)=a_{i}^{g}\left(P_{i}^{g}\right)^{2}+b_{i}^{g} P_{i}^{g}+c_{i}^{g}
$$

where $k_{c}$ is the fuel price, $a_{i}^{g}=k_{c, i} a_{i}^{F}, b_{i}^{g}=k_{c, i} b_{i}^{F}$, and $c_{i}^{g}=k_{c, i} c_{i}^{F}$.

The emissions of a unit from fuel combustion are given by:

$$
E_{i}^{g}\left(P_{i}^{g}\right)=k_{e, i} F_{i}\left(P_{i}^{g}\right)=a_{i}^{e}\left(P_{i}^{g}\right)^{2}+b_{i}^{e} P_{i}^{g}+c_{i}^{e}
$$

where $k_{e}$ is the emission factor of the unit, $a_{i}^{e}=k_{e, i} a_{i}^{F}, b_{i}^{e}=k_{e, i} b_{i}^{F}$, and $c_{i}^{e}=k_{e, i} c_{i}^{F}$. We also assume that renewable generation has no fuel cost or emissions.

\subsection{Simulation setup}

The analyses of impacts of adding energy storage-based reserves are carried out for a variety of system configurations. As briefly mentioned in Section 2.1, to evaluate the $\mathrm{CO}_{2}$ emissions of a given system, we solve the OPF problem (1) - (9) to obtain the dispatch schedule, and then calculate the $\mathrm{CO}_{2}$ emissions for each traditional generator using (17). The sum of emissions from all units is the total system emissions.

In our simulation study, we first define a base case. Then, several parameters are varied and the results are analyzed and compared with the base case. The analysis is also conducted for the IEEE 30-bus system [24, 25] with 6 traditional generators and 41 transmission lines. Reformulations of the $\mathrm{OPF}$ problem to incorporate reserves in transmission line constraints and $\mathrm{CO}_{2}$ emissions are also investigated.

In the base case, the following parameters are used:

1. Loads are scaled to a total of $700 \mathrm{MW}$.

2. Fuel costs data from the Energy Information Administration (EIA) for the year of 2013 are used: $k_{c, \text { coal }}=\$ 2.3 / \mathrm{MMBtu}, k_{c, \text { gas }}=\$ 4.3 / \mathrm{MMBtu}[40]$. The full load heat rates are 12.6 MMBtu/MWh for coal units and 9.0 MMBtu/MWh for gas units. More details about the fuel characteristics and generation costs can be found in Section 2.4. 
3. Reserve costs for Coal 1, Coal 2, Gas 1, Gas 2 are $\$ 10 / \mathrm{MW}, \$ 9 / \mathrm{MW}, \$ 5 / \mathrm{MW}$, and $\$ 4 / \mathrm{MW}$, respectively. In [41], it is assumed that the operational cost for reserves is $\$ 10 / \mathrm{MW}$ for coal units and $\$ 6 / \mathrm{MW}$ for natural gas units. In this paper, we adopt similar numbers and neglect opportunity costs.

4. Emission factors data from the EIA for the year of 2013 are used: $k_{e, \text { coal }}=210 \mathrm{lb} / \mathrm{MMBtu}$, $k_{e, \text { gas }}=117 \mathrm{lb} / \mathrm{MMBtu}[40]$.

5. The round trip efficiency $\beta$ for energy storage is set to 0.7 , which is typical for several common batteries [42]. In estimating the impacts of the round trip loss, we used the PJM dynamic regulation signal (RegD) on May 4, 2014 [43]. For that 24-hour period, the coefficient $k^{E S}$ is calculated to be $k^{E S}=3.046$. From (11), we get the additional load $P^{d, E S}=0.038 R^{E S}$.

6. The system is not congested in the base case; the congested case is discussed in Section 3.2.

7. There are no emissions caused by reserves actions in the base case; the impacts of emissions from reserves are discussed in Section 3.2.

In each case, we run two sets of simulations. First, since energy storage reduces the need for traditional operating reserves, it is helpful to understand how the reserve requirement affects the power system without energy storage. As the amount of reserves required varies, the system dispatch changes accordingly. The dispatch results and system emissions for different reserve requirements are presented; see, for example, the left side of Figure 4 (which will be fully described in Section 3.1).

In our second set of simulations we include the energy storage, providing more accurate results. The results are presented in contour plots, which show the difference in system $\mathrm{CO}_{2}$ emissions after we add energy storage; see, for example, the right side of Figure 4 (which will be fully described in Section 3.1). Positive values indicate an increase in emissions and negative values indicate a reduction. If we fix a reserve requirement value and move along the available energy storage axis, we observe the emissions impacts of adding different amounts of energy storage. For example, in Figure 4, if the system requires $150 \mathrm{MW}$ of reserves, having a small amount of energy storage reduces the system emissions; however, beyond $100 \mathrm{MW}$, adding more energy storage increases the system emissions.

There are several commonly used rules to determine reserve requirements in power systems. 
One rule is to require reserves to be no less than a certain fraction of the forecasted peak load. Another is the $3+5$ rule, which requires reserves to be at least $3 \%$ of the forecasted load plus $5 \%$ of the forecasted renewable generation [3]; this translates to $31 \mathrm{MW}$ in the base case. Recent studies also proposed frameworks to make the requirement a decision variable and determine it together with the dispatch in the OPF problem; see, for example, [44]. In this paper, we investigate how different reserve requirements affect the environmental outcome of adding energy storage. The reserve requirement from the $3+5$ rule are marked in our plots for reference.

\subsection{Overview of Scenarios}

To carefully study the impacts of adding energy storage-based reserves and what factors affect the results, we first simulate the base case; then we study the following three scenarios and compare them with the base case: S1, where renewable energy production is curtailed due to reserve requirements; S2, where there is flexibility from traditional generators to provide reserves without changing their generation schedule; S3, where the conditions in S1 and S2 do not apply. A summary of these scenarios are shown in Figure 3 and detailed descriptions will be presented in the following section.

\section{Results and discussion}

\subsection{Base case}

The results for the two sets of simulations are shown in Figure 4. We see that as the reserve requirement increases, the system $\mathrm{CO}_{2}$ emissions could increase, remain the same, or decrease. More analyses on these results are presented in comparison with other cases in Section 3.2.

\subsection{Three scenarios}

The following scenarios correspond to those in Figure 3. 


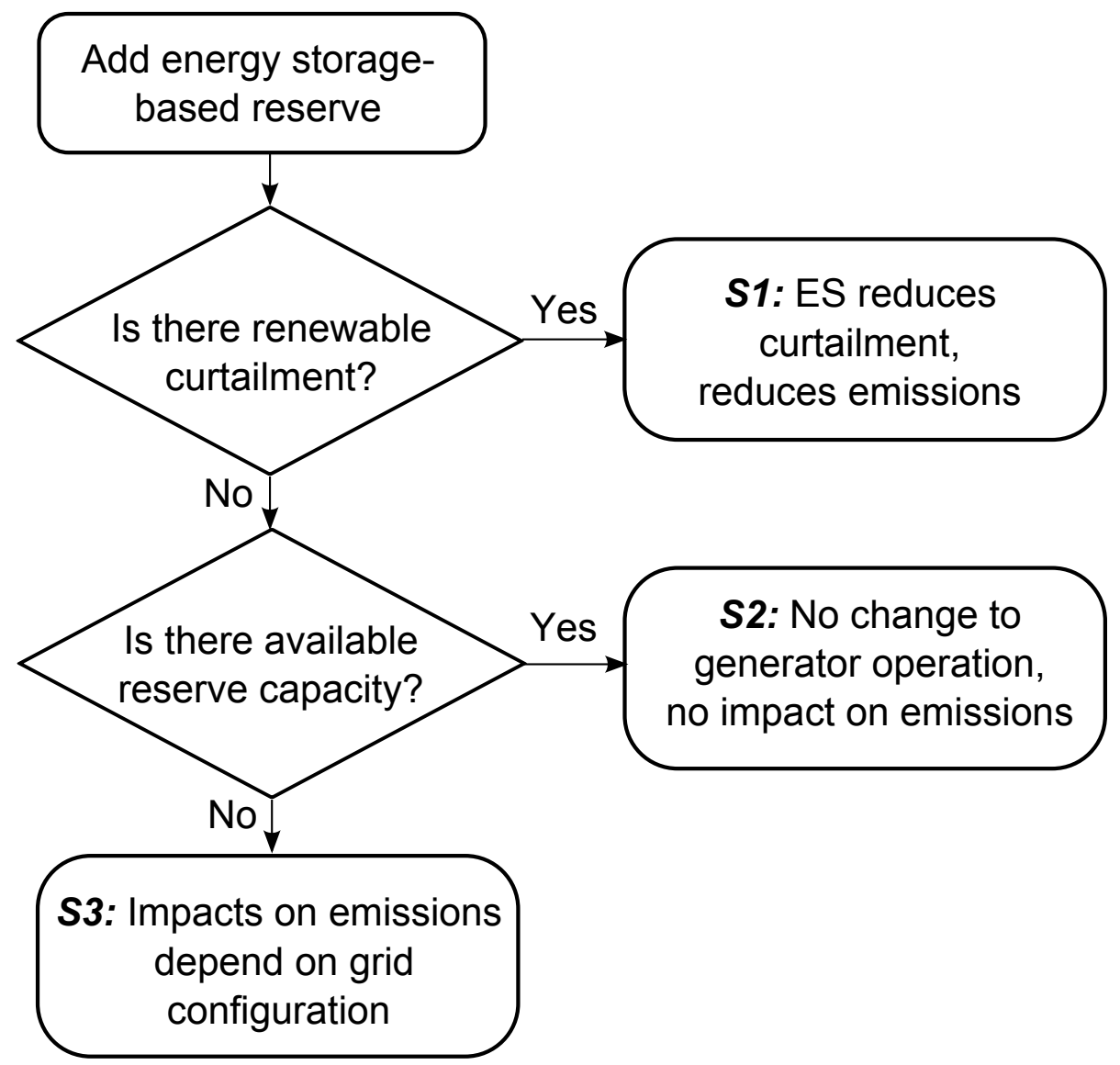

Figure 3: Summary of emissions impacts of energy storage-based reserves in different scenarios. ES refers to energy storage. 


\subsubsection{S1: with renewable curtailment}

There are several causes for renewable curtailment, one of which is reserve requirements. During periods where load is low and renewable generation is high, the traditional generators may operate close to their minimum outputs. However, when required to provide downward reserve, the generators have to increase their output so that there is capacity to ramp down. This leads to higher than desired generation from the traditional generators relative to the load and renewable generation, yielding renewable curtailment.

In this case, if energy storage is available for reserves, the burden of providing downward reserve could be shifted to the energy storage devices, and traditional generators could operate closer to minimum outputs; thus more renewable generation can be utilized. After adding energy storage, part of the traditional generation is replaced by renewable energy, which has almost no emissions, leading to a reduction in the system emissions, which outweighs any emissions increases energy storage may cause in power system operation. An example of this scenario is shown in Figure 4, when reserve requirement is in the range of 100 to $200 \mathrm{MW}$. As shown in the plot, when the reserve requirement increases, traditional generators have to increase their output to provide reserves, leading to more curtailment of renewable generation. In this case, adding energy storage-based reserves helps the power system use more renewable energy, thus reducing $\mathrm{CO}_{2}$ emissions.

\subsubsection{S2: with available reserve capacity}

When a generator is operating at partial load (not already being used for reserves), it could provide reserves without changing its power generation. In this case, we say it has available reserve capacity. When such a unit is the marginal unit for providing reserves, adding energy storage-based reserves reduces the reserve capacity the unit provides, but does not change the power generation of the unit. Thus it has no impact on system emissions. This behavior can be observed from Figure 4, when the reserve requirement is in the range of 50 to $80 \mathrm{MW}$. The generator Coal 2 operates at $150 \mathrm{MW}$ and has $30 \mathrm{MW}$ of downward available reserve capacity and $150 \mathrm{MW}$ of upward available reserve capacity. So when the reserve requirement is less than $30 \mathrm{MW}$, there is no change in its generation since the available reserve capacity is sufficient to cover the reserve requirement. Thus, 
in this scenario, adding energy storage-based reserves does not have any impact on emissions.

\subsubsection{S3: general cases}

If the conditions in S1 and S2 do not apply, the generators have to change their generation schedule to provide the reserves required. This is shown in Figure 4, when the reserve requirement is less than $50 \mathrm{MW}$ or in the range of 80 to $100 \mathrm{MW}$.

In this scenario, determining the impacts of adding energy storage is more complex. There are two layers in the problem. First, depending on the system configuration (generator/reserve costs, load level, transmission congestion, and etc.), adding energy storage could result in a change in generation allocation - we call this layer of the problem $Q_{1}$; second, depending on the generator characteristics, a change in generation allocation could result in different impacts on the system emissions - we call this layer of the problem $Q_{2}$.

For $Q_{1}$, we investigate several important factors in the system configuration that affect the dispatch results.

Generation and reserve costs. The objective of solving the OPF problem is to minimize the system generation and reserve cost, so the cost characteristics for each generator directly affects the dispatch result. The cost difference between generators, as well as the cost difference between generation and reserves, play a more important role than the actual value of the cost. This is because the ordering of units' cost, not the actual values of costs, determines the dispatch results. For instance, consider the situation where one generator is operating at partial load and others are operating at their limits. The marginal cost of using the partial load unit to provide reserves is simply its reserve cost; we call it $c_{\text {opt } 1}$. If we want to use another unit to provide reserves, it would have to shift its generation due to its generation limit. The cost is then the sum of the reserve cost of that unit and the generation cost difference caused by the shift; we call it $c_{\text {opt } 2}$. Either option could be better depending on the particular cost characteristics. As a result, the emissions impacts of adding energy storage-based reserves are also affected. For example, in the base case in Figure 4, when the reserve requirement is less than $50 \mathrm{MW}$, generator Coal 1 operates at partial load. However, $c_{o p t 1}$ for using Coal 1 is greater than $c_{o p t 2}$ for using Gas 2. Thus, instead of using the available reserve capacity of 
Coal 1, Gas 2 is dispatched for reserves first. Since Gas 2 is operating at its limit, the generation allocation has to change. If we change the reserve cost for Coal 1 to a lower value, so that $c_{o p t 1}$ for using Coal 1 is less than $c_{o p t 2}$ for using any other generator, then the available reserve capacity of Coal 1 would be dispatched first, and the generation allocation would remain the same; this result is shown in Figure 5. If natural gas prices become lower than coal prices, energy storage-based reserves would generally have positive or neutral emissions impacts, since they would be unlikely to increase coal generation, which is the major cause for the emissions increases observed here.

Load level. The power balance equations (4) ensures that all load is met. Thus, the load level directly affects the generation output. This, in turn, affects the units' capacity to provide reserves. Different load levels lead to different generator operation, which causes different emissions impacts related to adding energy storage-based reserves. One example is shown in Figure 6, where the only difference is the load level is set at $800 \mathrm{MW}$ instead of $700 \mathrm{MW}$, which is used in the base case. Note that if the system includes base load generators, such as nuclear power plants, which do not vary their outputs often or provide reserves, they would have a similar effect on the results as reducing the system load.

Transmission line constraints. Transmission line constraints limit the power flow on a line, which could prevent the most cost-efficient energy from being delivered to the load. Figure 7 shows the results with a binding line constraint for the line from bus 6 to 7 . When the reserve requirement is larger than $130 \mathrm{MW}$, the line constraint becomes binding, preventing Gas 2 from increasing its generation to provide more reserves. When the reserve requirement exceeds $180 \mathrm{MW}$, there is no longer any feasible dispatch that satisfies all constraints.

Round trip loss. As shown in (11), both poor round trip efficiency and large $k^{E S}$ cause large round trip losses. The resulting additional load increases the cost of using energy storage for reserves, and energy storage devices become less attractive, in terms of both system cost and emissions. In the extreme case, it is better to just use traditional generators to provide reserves even if energy storage is available. One example of such case is shown in Figure 8 , where $\beta=0.4, k^{E S}=12$, 
and there are $50 \mathrm{MW}$ of energy storage-based reserves available. We observe that when the reserve requirement is less than $30 \mathrm{MW}$, Gas 2 is dispatched first for reserves instead of the energy storage.

Emissions from traditional generator reserves. When reserves are called, generator outputs vary accordingly to balance supply-demand mismatch. While the resulting ramping may result in additional emissions, the amount is not well understood. For example, in [6], it is reported that only 76-79\% of expected $\mathrm{CO}_{2}$ reduction from wind generation can be achieved if natural gas units are used to provide reserves; in [45], the number is $94 \%$; in [5], the authors conclude the impacts of ramping on $\mathrm{CO}_{2}$ emissions are negligible.

Including the emissions due to traditional generator reserves changes the total system emissions, but does not change the dispatch (unless we include an emissions constraint, as in Section 3.5). This gives energy storage-based reserves additional emissions reduction benefits compared to the base case. From (17), we know the $\mathrm{CO}_{2}$ emissions rate $r_{\mathrm{CO}_{2}}$ of a traditional generator is:

$$
r_{C O_{2}, i}\left(P_{i}^{g}\right)=\frac{d}{d P_{i}^{g}} E_{i}^{g}\left(P_{i}^{g}\right)=2 a_{i}^{e} P_{i}^{g}+b_{i}^{e}
$$

We calculate $r_{\mathrm{CO}_{2}}$ for each traditional generator operating at its scheduled output, and we let the emissions from its reserves be:

$$
E_{i}^{r}\left(R_{i}^{g}\right)=\gamma r_{C O_{2}, i} R_{i}^{g}
$$

where $\gamma$ is the coefficient for reserve emissions. We tested different values for $\gamma$, from zero to 0.25 . The results when $\gamma=0.25$ are shown in Figure 9. Comparing these results with those of the base case, energy storage-based reserves lead to more favorable emissions outcomes.

Now we look at the second layer of the problem, $Q_{2}$. Different generators usually have different heat rates and emission factors, so a change in generation allocation leads to a change in emissions. Here, we demonstrate this behavior in an example where the allocation is changed between two generators and the fuel consumed is quadratic with respect to the power generation.

Suppose the emissions of a unit are given by (17). Consider two generators with a total load 
level of $d$, we have:

$$
P_{1}^{g}+P_{2}^{g}=d
$$

The total emissions of the two generators can be expressed in terms of $P_{1}^{g}$ :

$$
\begin{aligned}
E^{g}= & \left(k_{e, 1} a_{1}^{e}+k_{e, 2} a_{2}^{e}\right) P_{1}^{g 2}+\left(k_{e, 1} b_{1}^{e}-k_{e, 2} b_{2}^{e}-2 k_{e, 2} a_{2}^{e} d\right) P_{1}^{g} \\
& +\left(k_{e, 1} c_{1}^{e}+k_{e, 2} c_{2}^{e}+k_{e, 2} a_{2}^{e} d^{2}+k_{e, 2} b_{2}^{e} d\right)
\end{aligned}
$$

The total emissions are quadratic with respect to $P_{1}^{g}$, and there is a minimum at:

$$
P_{1}^{g *}=\frac{-\left(k_{e, 1} b_{1}^{e}-k_{e, 2} b_{2}^{e}-2 k_{e, 2} a_{2}^{e} d\right)}{2\left(k_{e, 1} a_{1}^{e}+k_{e, 2} a_{2}^{e}\right)}
$$

We see that the directional emissions impacts depend not only the fuel characteristics, but also on the operating point. A very special case is when the two units have the same emission factors and fuel characteristics. The minimum emissions are then achieved at:

$$
P_{1}^{g *}=d / 2
$$

In this example, if $P_{1}^{g}$ is less than $P_{1}^{g *}$, increasing $P_{1}^{g}$ reduces emissions; but when $P_{1}^{g}$ is greater than $P_{1}^{g *}$, the impact is the opposite.

\subsection{0-bus test system}

We also evaluate the emissions impacts of energy storage-based reserves on a more complex 30-bus test system $[24,25]$ to gain additional insights. A renewable energy source of $50 \mathrm{MW}$ is added to bus 28. An energy storage device is also added to bus 28. Loads are scaled by a factor of 1.1 to introduce congestion into the system. Simulation results are shown in Figure 10.

Compared to the 9-bus system, the 30-bus system has more generators and loads and a more complex topology. However, we observed several behaviors in the 30 -bus system similar to those in the simple 9-bus system. For reserve requirements of less than $30 \mathrm{MW}$, there is available reserve 
capacity from the traditional generators, thus adding energy storage has no impacts on system emissions - similar to S2; for reserve requirements larger than $80 \mathrm{MW}$, adding energy storage helps reduce renewable curtailment, which reduces the system emissions - similar to S1; however, due to the complexity of the system, there are non-trivial and non-intuitive behaviors over other ranges of reserve requirements - similar to $\mathrm{S} 3$.

In both the 9-bus and the 30-bus systems, we have a single renewable source and a single energy storage device. Having multiple smaller renewable power plants and multiple storage systems could reduce renewable curtailment and congestion [46]. In such grids, the analyses in S2 and S3 are more relevant.

\subsection{Impacts of considering reserves in line flow constraints}

In the previous sections, only the scheduled power flow from generation was considered when computing transmission line constraints. However, when reserves are called, the actual generation is different from the schedule, and additional power may flow through transmission lines violating the transmission line constraints. In this section, we present an approach to include the flow from reserve actions in the transmission line constraints. We observe that this change in formulation also affects the emissions impacts of energy storage-based reserves.

We assume that the power flow through each line has to be under the limit, not only in the 
scheduled case, but also in all cases when reserves are used. The new OPF problem is:

$$
\begin{aligned}
& \underset{X}{\operatorname{minimize}} \quad f^{g}\left(P^{g}\right)+f^{r}\left(R^{g}\right)+f^{E S}\left(R^{E S}\right) \\
& \text { subject to } \quad P_{f}=B_{f} \Theta \\
& P_{b u s}=B_{b u s} \Theta \\
& P_{b u s}=C^{g} P^{g}-C^{d} P^{d}-C^{E S} P^{d, E S} \\
& \mathbf{1}^{T} R^{g}+\mathbf{1}^{T} R^{E S} \geq r_{r e q} \\
& P^{g}-R^{g} \succeq P_{\min }^{g} \\
& P^{g}+R^{g} \preceq P_{\max }^{g} \\
& R_{\min }^{E S} \preceq R^{E S} \preceq R_{\max }^{E S} \\
& P_{f, \min } \preceq P_{f} \preceq P_{f, \max } \\
& P_{f, \text { case } 1}=B_{f} \Theta_{\text {case } 1} \\
& P_{b u s, \text { case } 1}=B_{b u s} \Theta_{\text {case } 1} \\
& P_{\text {bus }, \text { case } 1}=C^{g} P_{\text {case1 }}^{g}-C^{d} P_{\text {case } 1}^{d}-C^{E S} P_{\text {case1 }}^{d, E S} \\
& P^{g}-R^{g} \preceq P_{\text {case } 1}^{g} \preceq P^{g}+R^{g} \\
& P_{f, \min } \preceq P_{f, \text { case } 1} \preceq P_{f, \max } \\
& \vdots \\
& P_{f, c a s e N}=B_{f} \Theta_{c a s e N} \\
& P_{b u s, \text { caseN }}=B_{b u s} \Theta_{\text {caseN }} \\
& P_{\text {bus }, \text { caseN }}=C^{g} P_{\text {caseN }}^{g}-C^{d} P_{\text {caseN }}^{d}-C^{E S} P_{\text {caseN }}^{d, E S} \\
& P^{g}-R^{g} \preceq P_{\text {caseN }}^{g} \preceq P^{g}+R^{g} \\
& P_{f, \min } \preceq P_{f, \text { caseN }} \preceq P_{f, \max }
\end{aligned}
$$

where $P_{\text {case } k}^{g}$ and $\Theta_{\text {case } k}$ are new variables that correspond to each case $k$ with different reserve 
Table 2: Direction of forecast deviations in four cases.

\begin{tabular}{ccc}
\hline & Renewable source & Loads \\
\hline Case 1 & + & + \\
Case 2 & + & - \\
Case 3 & - & + \\
Case 4 & - & - \\
\hline
\end{tabular}

realizations. Each set of additional constraints (for example, (33) to (37)) states that there must be a new generation dispatch within the range $\left[P^{g}-R^{g}, P^{g}+R^{g}\right]$ which handles the reserve realization and satisfies the transmission line constraints.

Reserves are required when load and/or renewable generation deviate from forecasted values, and we assume each has a maximum deviation. Since load is often related to outdoor temperature [47], we assume all load deviations from their forecasts have the same sign. We consider four extreme cases, where each forecast has the largest deviation possible in either the positive or negative direction; see Table 2. See [22] for a method of including an arbitrary number of cases, corresponding to different reserve realizations, within an OPF formulation. For demonstration, we assume the largest possible deviation for each load/renewable resource is $1 / 4$ of the total reserve requirement; for example, if the system requires $40 \mathrm{MW}$ of reserve, the renewable resources and loads each deviates by 10 MW. Figure 11 shows the comparison between the original OPF solution and the new formulation. We see that when the reserve requirement is moderate, there are no significant differences in the results; however, when there is substantial requirement for reserves, the dispatch could be significantly different.

\subsection{Impacts of a $\mathrm{CO}_{2}$ emissions cap}

As shown in the simulations above, adding energy storage-based reserves to a power system sometimes increases the total system $\mathrm{CO}_{2}$ emissions, which is undesirable. There are several approaches to include emissions in the dispatch algorithm; for example, adding emissions costs in the objective function [16-18] or adding emissions constraints [19]. Here, we study the dispatch results and costs when the system is constrained so that the emissions with energy storage are at most the emissions without energy storage. Thus, we add an additional constraint to the OPF. First, the 
original OPF problem without energy storage is solved and the system $\mathrm{CO}_{2}$ emissions $E_{\text {orig }}$ are computed. Then, a second OPF problem with energy storage and $\mathrm{CO}_{2}$ emissions constraint is solved to determine the dispatch. The additional $\mathrm{CO}_{2}$ constraints is:

$$
E^{g}\left(P^{g}\right) \leq E_{\text {orig }}
$$

From (17), the constraint can be expressed as:

$$
\sum_{i} a_{i}^{e} P_{i}^{g^{2}}+b_{i}^{e} P_{i}^{g}+c_{i}^{e} \leq E_{\text {orig }}
$$

A useful property of this constraint is that the left hand side is quadratic with respect to the variables $P_{i}^{g}$ and the less or equal relation makes it a convex constraint. Thus the new OPF problem is a convex quadratically constrained quadratic program (QCQP), which can be solved efficiently with established solvers. Figure 12 shows a comparison among different OPF formulations for the base case. Three cases are considered: (i) there is no energy storage, all reserves are provided by traditional generators; (ii) there is $50 \mathrm{MW}$ of energy storage available for reserves, but there is no constraint on system emissions; (iii) there is $50 \mathrm{MW}$ of energy storage available for reserves and a system emissions constraint. System emissions and cost are shown in Figure 12. We observe that there is a break point when the reserve requirement is $110 \mathrm{MW}$. Below that point, adding energy storage increases emissions (comparing (i) and (ii)). In (iii), the $\mathrm{CO}_{2}$ constraint prevents an increase in system emissions, thus the emissions are the same as in (i). The cost in (iii) is not as low as it is in case (ii); however, the cost is still lower than (i) because energy storage provides more flexibility which allows the system to find more efficient dispatch. Beyond the break point, adding energy storage decreases emissions (comparing (i) and (ii)), thus the $\mathrm{CO}_{2}$ constraint in (iii) is not binding and the dispatches in (ii) and (iii) are the same. This simulation demonstrates that in some cases, there can be a trade-off between cost and emissions when adding energy storage-based reserves to the system. 


\subsection{Policy implications}

From our simulations and analyses, we find that policies to incentivize energy storage may not reduce $\mathrm{CO}_{2}$ emissions. In recent years, the regulatory environment has been improving for energy storage, partially due to policy makers' belief that adding energy storage to the power system would lead to emissions reduction. For example, California's energy storage procurement mandate [11] claims that "Use of energy storage systems to provide the ancillary services otherwise provided by fossil-fueled generating facilities will reduce emissions of carbon dioxide and criteria pollutants." However, in contrast to this common view, our simulation results show that adding energy storagebased reserves may not necessarily reduce emissions in all power systems due to changes in dispatch; see, for example, in S2 and S3.

The results reported in this paper show that the emissions impacts of adding energy storage are significantly different in systems with different system configurations. Thus, policies encouraging energy storage should prioritize systems with configurations where larger emissions benefits could be achieved. To determine the expected emissions impacts in a particular system, an analysis like those in this paper could be conducted. The contour plots presented here are useful tools for helping policy makers determine whether or not energy storage would reduce or increase system emissions as a function of the amount of energy storage in the system and the reserve requirements. As our results show, determining whether system emissions will increase or decrease is complex, but emissions increases are more likely in systems with more expensive, flexible, low-emission generation on the margin and less expensive, non-flexible, high-emission generation providing the base load. Based on our findings, it would be reasonable to assume that ISOs with a sizable mix of coal and natural gas generation (e.g., MISO, PJM) would have a greater opportunity, and thus greater risk, of significant changes to system-wide emissions when using energy storage for reserves. In ISOs with limited reliance on coal, such as CAISO and ISO-NE, we would expect more modest changes in system emissions. However, the role of electricity imports could complicate the findings. To determine the impacts to a particular ISO, one would need to run a model for that ISO and perform analyses like those in this paper.

In areas where emissions are expected to increase, improving the dispatch algorithm to account 
for emissions, via emissions constraints or costs, is a way to ensure more favorable emissions outcomes. However, this may increase system costs, and emissions/cost trade-offs should be taken into consideration. 

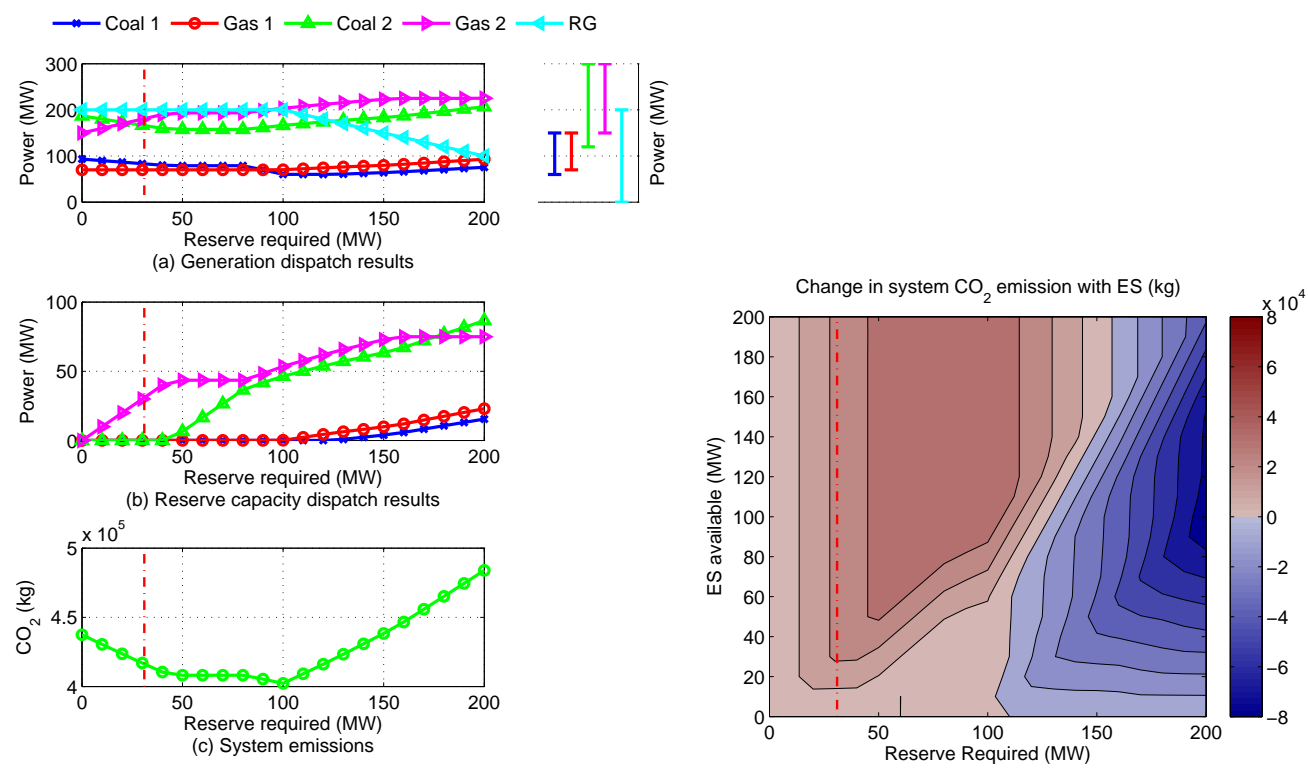

Figure 4: Simulation results in the base case. Left: (a) and (b) show the dispatch results, (c) shows the system emission; right: the contour plot shows the emissions impacts of adding energy storage-based reserves. Red colors indicate increases in system $\mathrm{CO}_{2}$ emissions and blue colors indicate reductions. The red vertical lines indicate the reserve requirement based on the $3+5$ rule. RG refers to renewable generation; ES refers to energy storage. 


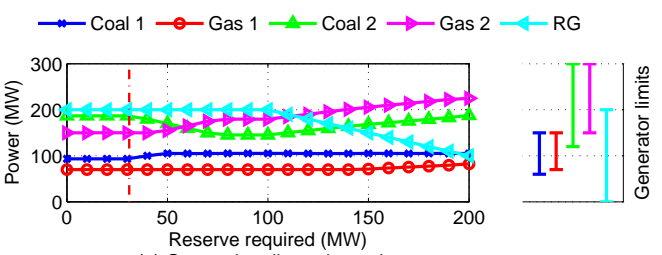

(a) Generation dispatch results

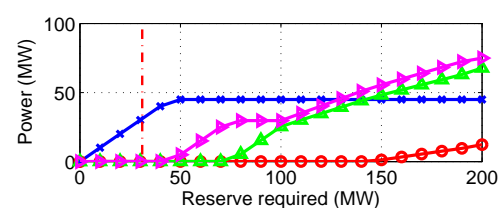

(b) Reserve capacity dispatch results
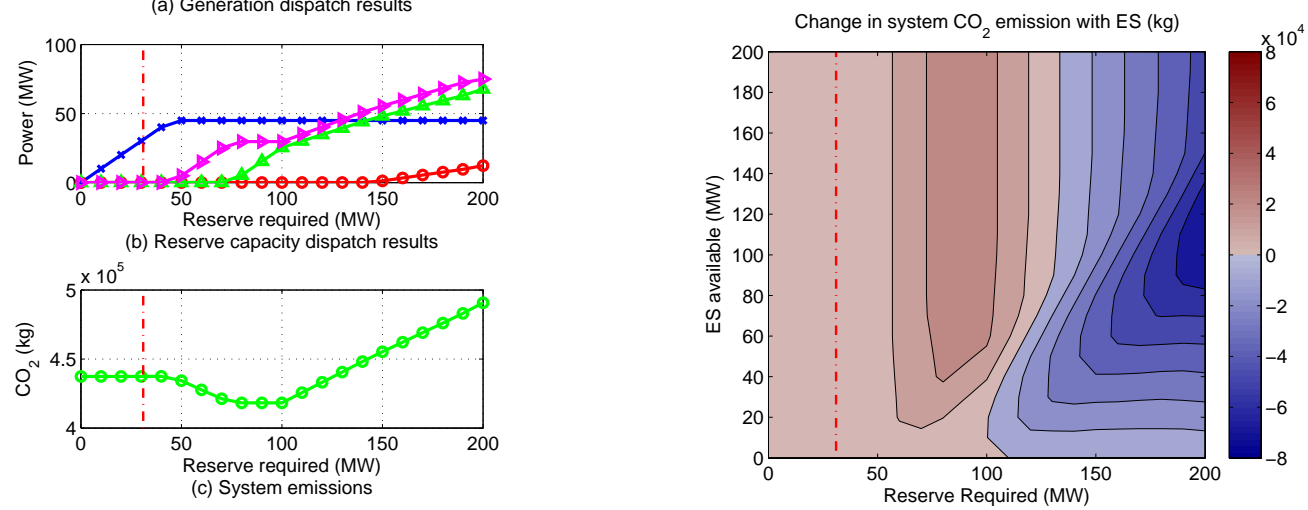

Figure 5: Simulation results in the case where reserve cost for Coal 1 is low compared to the base case. Left: (a) and (b) show the dispatch results, (c) shows the system emission; right: the contour plot shows the emissions impacts of adding energy storage-based reserves. Red colors indicate increases in system $\mathrm{CO}_{2}$ emissions and blue colors indicate reductions. The red vertical lines indicate the reserve requirement based on the $3+5$ rule. RG refers to renewable generation; ES refers to energy storage. 


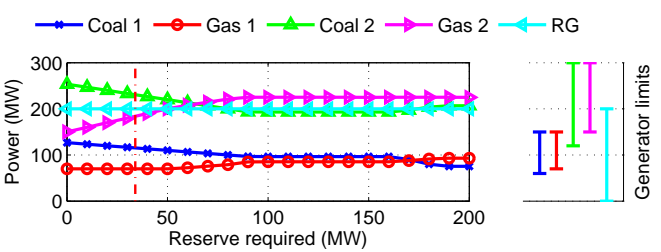

(a) Generation dispatch results

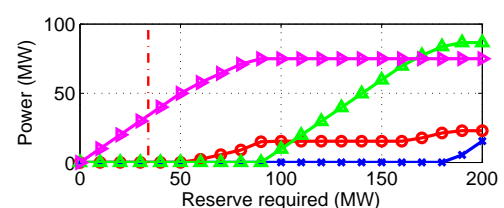

(b) Reserve capacity dispatch results

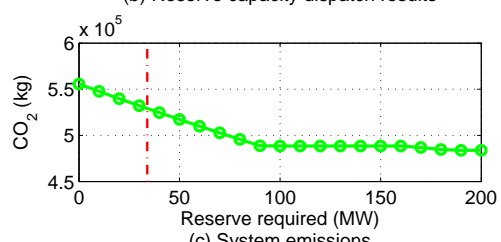

Change in system $\mathrm{CO}_{2}$ emission with $\mathrm{ES}(\mathrm{kg})$

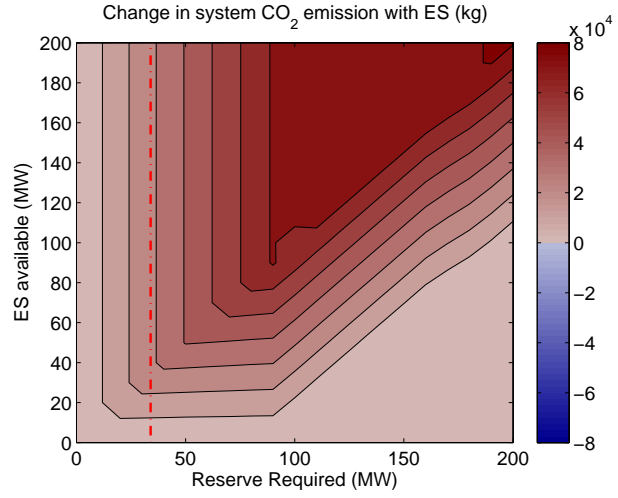

Figure 6: Simulation results in the case where load level is high compared to the base case. Left: (a) and (b) show the dispatch results, (c) shows the system emission; right: the contour plot shows the emissions impacts of adding energy storage-based reserves. Red colors indicate increases in system $\mathrm{CO}_{2}$ emissions and blue colors indicate reductions. The red vertical lines indicate the reserve requirement based on the $3+5$ rule. RG refers to renewable generation; ES refers to energy storage. 


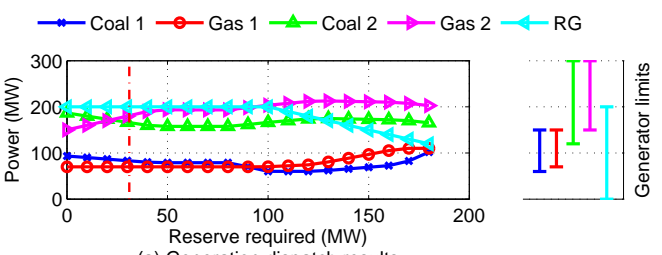

(a) Generation dispatch results
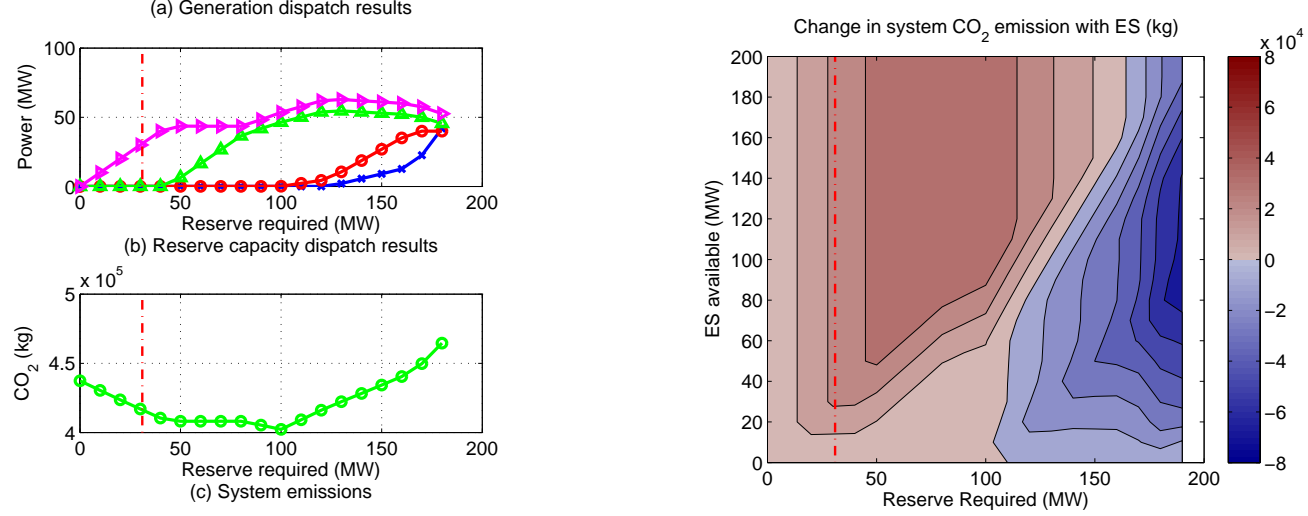

Figure 7: Simulation results in the case where transmission line from bus 6 to 7 has a power flow limit of 170 MW. Left: (a) and (b) show the dispatch results, (c) shows the system emission; right: the contour plot shows the emissions impacts of adding energy storage-based reserves. Red colors indicate increases in system $\mathrm{CO}_{2}$ emissions and blue colors indicate reductions. The red vertical lines indicate the reserve requirement based on the $3+5$ rule. RG refers to renewable generation; ES refers to energy storage. 


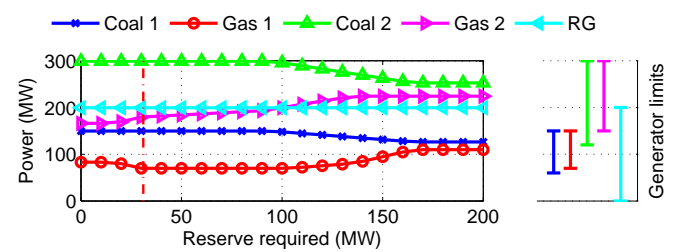

(a) Generation dispatch results

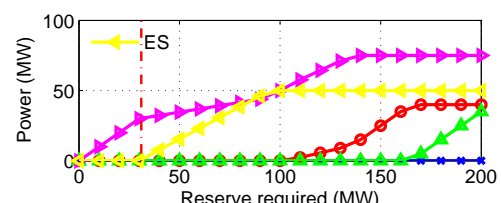

(b) Reserve capacity dispatch results
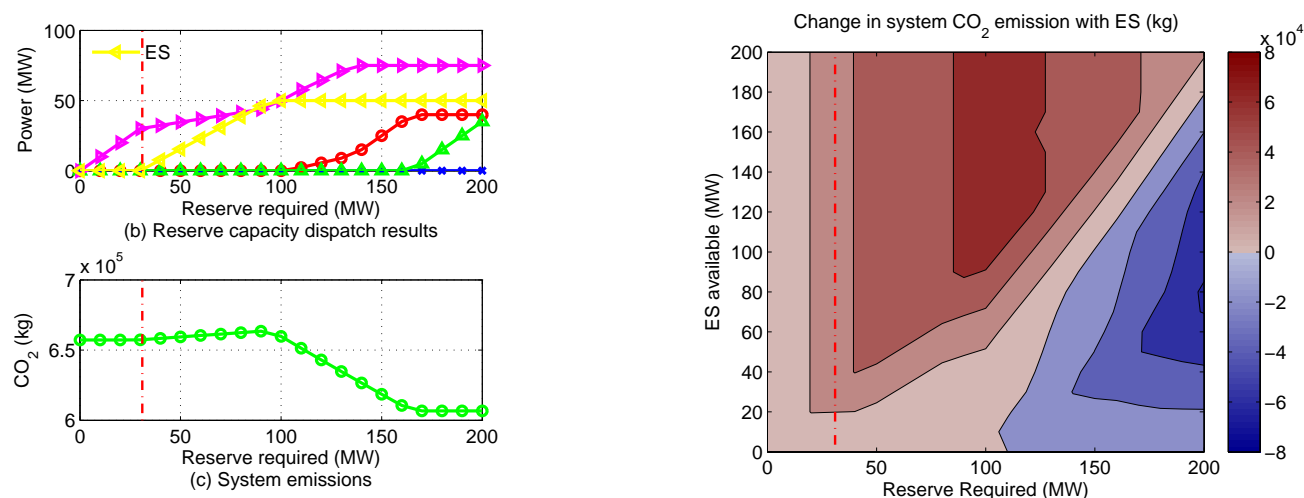

Figure 8: Simulation results in the case where round trip loss from energy storage is large compared to the base case. Left: (a) and (b) show the dispatch results, (c) shows the system emission; right: the contour plot shows the emissions impacts of adding energy storage-based reserves. Red colors indicate increases in system $\mathrm{CO}_{2}$ emissions and blue colors indicate reductions. The red vertical lines indicate the reserve requirement based on the $3+5$ rule. $\mathrm{RG}$ refers to renewable generation; ES refers to energy storage. 


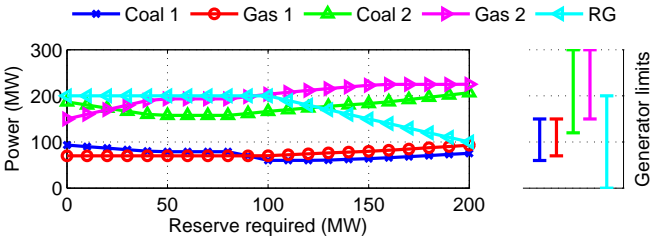

(a) Generation dispatch results

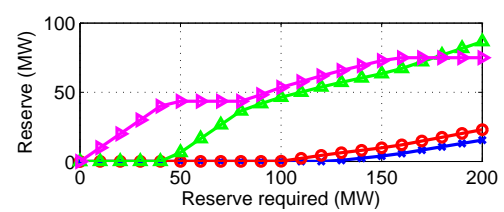

(b) Reserve capacity dispatch results
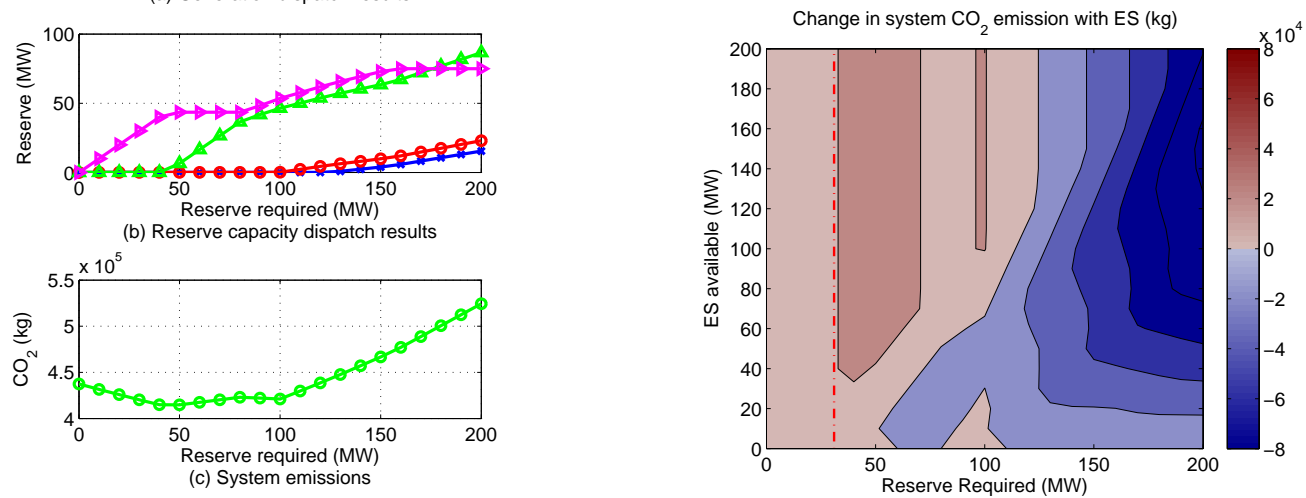

Figure 9: Simulation results with emissions for traditional generator reserves where $\gamma=0.25$. Left: (a) and (b) show the dispatch results, (c) shows the system emission; right: the contour plot shows the emissions impacts of adding energy storage-based reserves. Red colors indicate increases in system $\mathrm{CO}_{2}$ emissions and blue colors indicate reductions. The red vertical lines indicate the reserve requirement based on the $3+5$ rule. RG refers to renewable generation; ES refers to energy storage. 

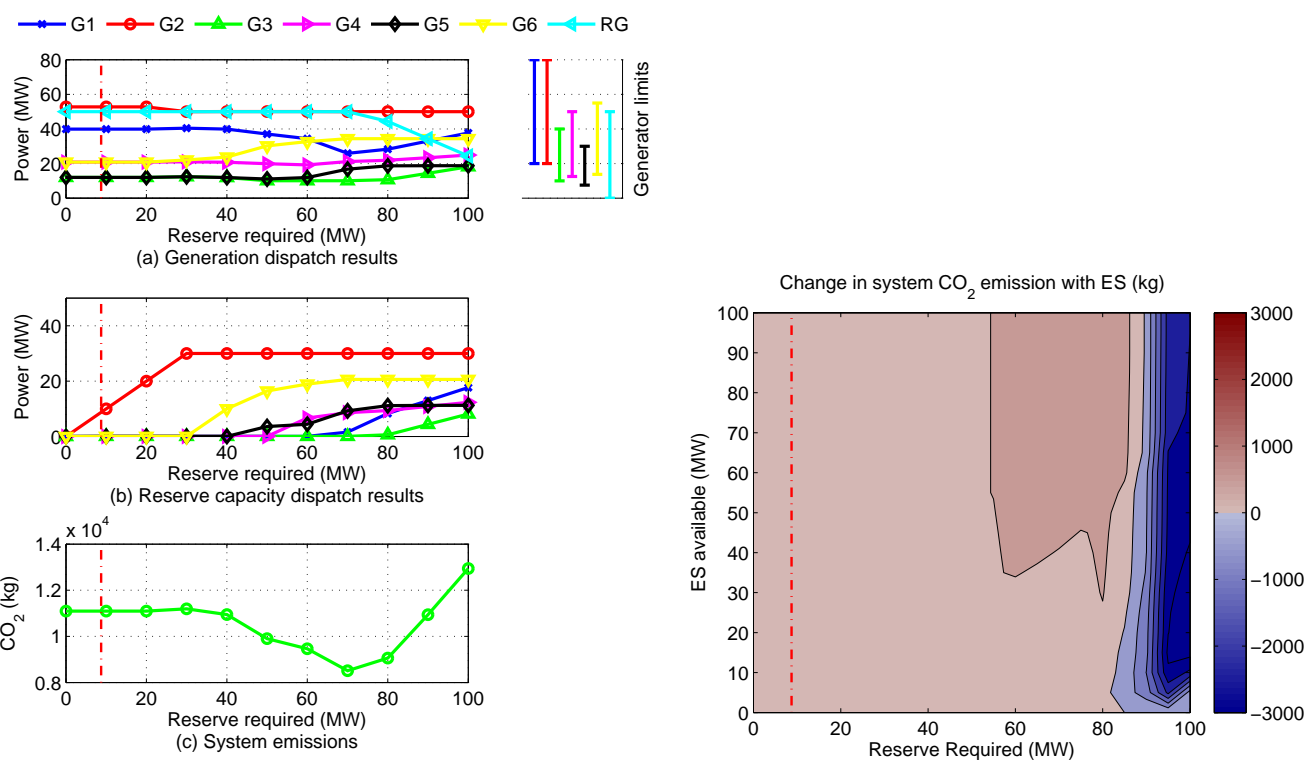

Figure 10: Simulation results for the modified IEEE 30-bus system. Left: (a) and (b) show the dispatch results, (c) shows the system emission; right: the contour plot shows the emissions impacts of adding energy storage-based reserves. Red colors indicate increases in system $\mathrm{CO}_{2}$ emissions and blue colors indicate reductions. The red vertical lines indicate the reserve requirement based on the $3+5$ rule. RG refers to renewable generation; ES refers to energy storage. 


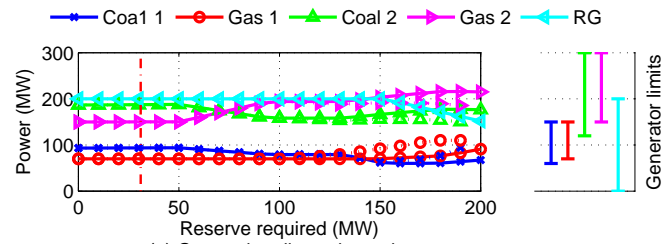

(a) Generation dispatch results

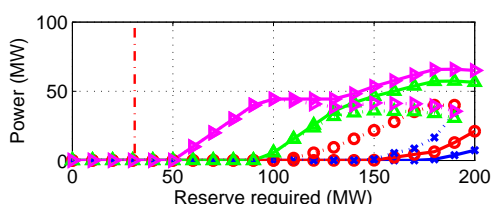

(b) Reserve capacity dispatch results

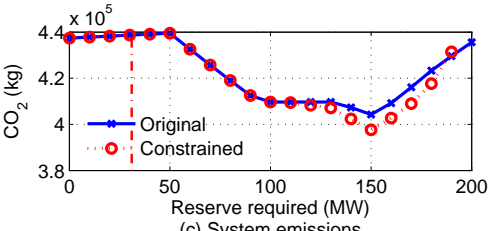

(c) System emissions

Figure 11: Impacts of including maximum possible reserve realizations in transmission line constraints. Solid lines are results for the case where reserves are not considered in transmission line flow constraints; dotted lines are results for the case where they are included. The red vertical lines indicate the reserve requirement based on the $3+5$ rule. 

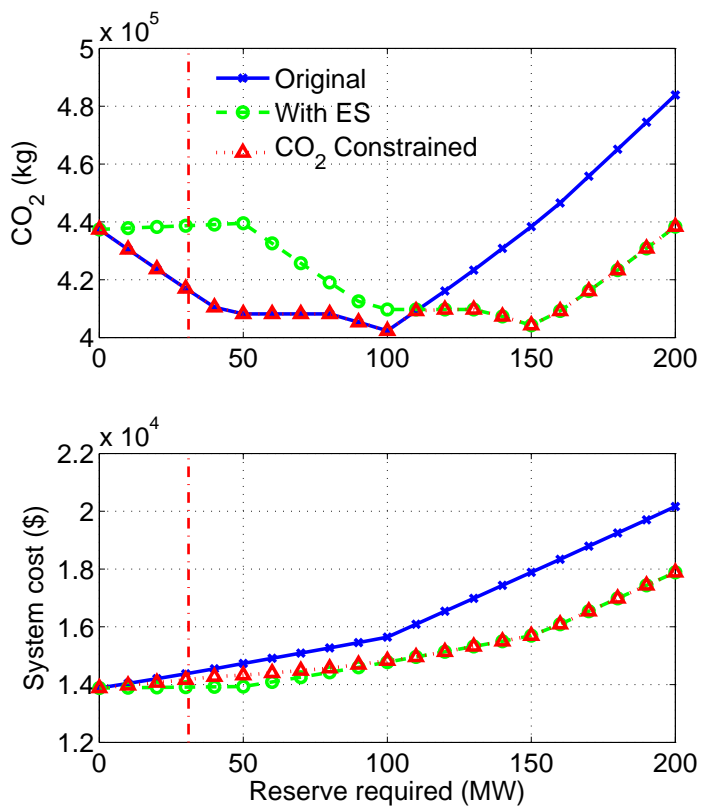

Figure 12: System emissions and cost in three different OPF formulations. Solid lines are for the original case without energy storage; dashed lines are for the case with $50 \mathrm{MW}$ of energy storage available and no $\mathrm{CO}_{2}$ constraint; dotted lines are for the case with energy storage and a $\mathrm{CO}_{2}$ constraint. The red vertical lines indicate the reserve requirement based on the $3+5$ rule. 


\section{Conclusion}

We investigate the impacts of adding energy storage-based reserves to a power system by solving OPF problems. Simulation results demonstrate that the emissions impacts of energy storage are significantly different for power systems with different system configurations. A few important factors are generation/reserve cost, load level, reserve requirement, the amount of energy storagebased reserve available, and transmission line constraints. In some scenarios, reserves provided by energy storage help renewable sources (such as wind) replace more traditional generation, reducing total emissions. In other scenarios, energy storage-based reserves do not impact renewable energy production levels; only the operation of traditional generators is affected, and the net emissions impacts could be either positive or negative. We also investigate reformulations of the classic OPF problem to explicitly limit emissions in the dispatch algorithm.

The results reported in this paper show that an insufficient understanding of the emissions impacts of using energy storage to provide reserves in the power system may lead to unreasonable assumptions in policies. The common view, adopted in the California's energy storage procurement mandate [11], that using energy storage for ancillary service reduces system emissions, may not hold true for all power systems. Depending on system configurations, adding energy storagebased reserves could cause a reduction, an increase, or no change in system emissions. Thus, a thorough emissions impacts evaluation is important in developing policies that achieve the desired environmental and economic objectives. To better utilize energy storage to reduce emissions, regions where emissions could be significantly reduced should be prioritized; for example, systems with substantial renewable curtailment, where energy storage would help reduce the curtailment.

Modifying the dispatch algorithm to incorporate emissions is an important step in achieving emissions goals. The underlying reason for non-intuitive emissions impacts is that the classic OPF formulation does not explicitly include emissions. In this paper, we present one possible reformulation: adding a $\mathrm{CO}_{2}$ constraint to the $\mathrm{OPF}$. By reformulating the OPF problem, the emissions impacts of different dispatches are explicitly represented in the optimization problem. Other possible reformulations, such as adding an emissions penalty to the cost function, are possible. 


\section{Acknowledgements}

This work was conducted with financial support from the National Science Foundation Environmental Sustainability Program award number 1510788, and the University of Michigan Energy Institute. 


\section{List of variables}

$\begin{array}{ll}\beta & \text { Energy storage round trip efficiency } \\ \gamma & \text { Traditional generator reserve emissions coefficient } \\ \Theta & \text { Bus voltage angle } \\ a^{e}, b^{e}, c^{e} & \text { Fuel combustion emissions coefficients } \\ a^{g}, b^{g}, c^{g} & \text { Generation cost coefficients } \\ B_{b u s} & \text { Power injection susceptance matrix } \\ B_{f} & \text { Power flow susceptance matrix } \\ C^{g}, C^{d}, C^{E S} & \text { Grid topology matrices } \\ d & \text { Total demand in the } Q_{2} \text { example } \\ E^{g} & \text { Emissions from power generation } \\ E_{\text {orig }} & \text { System emissions without energy storage } \\ E^{r} & \text { Emissions from reserves } \\ F & \text { Fuel consumed by generator } \\ f^{E S} & \text { Energy storage reserve cost } \\ f^{g} & \text { Generation cost } \\ f^{r} & \text { Reserve cost } \\ H & \text { Average heat rate } \\ k_{c} & \text { Fuel price } \\ k_{e} & \text { Emission factor } \\ k^{E S} & \text { Energy injected into energy storage per unit of reserve procurement } \\ L & \text { Energy storage round trip losses } \\ P_{b u s} & \text { Bus power injection } \\ P^{d} & \text { Load } \\ P^{d, E S} & \text { Load due to energy storage round trip losses } \\ P_{f} & \text { Transmission line power flow } \\ P^{g} & \text { Power generation } \\ r_{C O_{2}} & \text { Emission rate from traditional generator reserves } \\ R^{E S} & \text { Reserves from energy storage } \\ R^{g} & \text { Reserves from traditional generators } \\ r_{r e q} & \text { Reserve requirement } \\ W^{E S} & \text { Energy injected into energy storage due to reserves }\end{array}$




\section{References}

[1] D. A. Halamay, T. K. Brekken, A. Simmons, S. McArthur, Reserve requirement impacts of large-scale integration of wind, solar, and ocean wave power generation, Sustainable Energy, IEEE Transactions on 2 (3) (2011) 321-328.

[2] Y. V. Makarov, C. Loutan, J. Ma, P. de Mello, Operational impacts of wind generation on california power systems, Power Systems, IEEE Transactions on 24 (2) (2009) 1039-1050.

[3] GE Energy, Western wind and solar integration study, Tech. Rep. NREL/SR-550-47434, NREL (2010).

[4] E. Ela, M. Milligan, B. Kirby, Operating reserves and variable generation, Tech. Rep. NREL/TP-5500-51978, NREL (2011).

[5] D. Lew, G. Brinkman, E. Ibanez, B. Hodge, J. King, Western wind and solar integration study phase 2, Tech. Rep. NREL/TP-5500-55588, NREL (2013).

[6] W. Katzenstein, J. Apt, Air emissions due to wind and solar power, Environmental Science \& Technology 43 (2) (2008) 253-258.

[7] X. Luo, J. Wang, M. Dooner, J. Clarke, Overview of current development in electrical energy storage technologies and the application potential in power system operation, Applied Energy 137 (2015) 511-536.

[8] P. Denholm, J. Jorgenson, M. Hummon, T. Jenkin, D. Palchak, B. Kirby, O. Ma, M. O'Malley, The value of energy storage for grid applications, Tech. Rep. NREL/TP-6A20-58465, NREL (2013).

[9] Federal Energy Regulatory Commission, Order No. 755, Frequency regulation compensation in the wholesale power markets: Comments of ISO/RTO council.

[10] Federal Energy Regulatory Commission, Order No. 784, Third-party provision of ancillary services; accounting and financial reporting for new electric storage technologies. 
[11] California Public Utilities Commission, Order instituting rulemaking pursuant to Assembly Bill 2514 to consider the adoption of procurement targets for viable and cost-effective energy storage systems (October 2013).

[12] R. Sioshansi, P. Denholm, Emissions impacts and benefits of plug-in hybrid electric vehicles and vehicle-to-grid services, Environmental Science \& Technology 43 (4) (2009) 1199-1204.

[13] R. Sioshansi, P. Denholm, The value of plug-in hybrid electric vehicles as grid resources, Energy Journal 31 (3) (2010) 1-23.

[14] A. J. Wood, B. F. Wollenberg, Power generation, operation, and control, John Wiley \& Sons, 2012.

[15] A. R. Bergen, V. Vittal, Power systems analysis, Prentice Hall, 1999.

[16] P. Y. Kerl, W. Zhang, J. B. Moreno-Cruz, A. Nenes, M. J. Realff, A. G. Russell, J. Sokol, V. M. Thomas, New approach for optimal electricity planning and dispatching with hourly time-scale air quality and health considerations, Proceedings of the National Academy of Sciences 112 (35) (2015) 10884-10889.

[17] M. Shao, W. Jewell, $\mathrm{CO}_{2}$ emission-incorporated ac optimal power flow and its primary impacts on power system dispatch and operations, in: Power and Energy Society General Meeting, 2010 IEEE, IEEE, 2010, pp. 1-8.

[18] A. Jubril, O. Olaniyan, O. Komolafe, P. O. Ogunbona, Economic-emission dispatch problem: A semi-definite programming approach, Applied Energy 134 (2014) 446-455.

[19] R. Ramanathan, Emission constrained economic dispatch, Power Systems, IEEE Transactions on 9 (4) (1994) 1994-2000.

[20] California ISO, Non-generator resource regulation energy management project implementation plan, http://www.caiso.com/Documents/NonGeneratorResourceRegulationEnergyManagementImplementationPlan.pdf (March 2012). 
[21] NYISO, Energy Storage in the New York Electricity Market, http://www.nyiso.com/ public/webdocs/markets_operations/committees/bic_miwg/meeting_materials/201001-21/Energy_Storage_Resources.pdf.

[22] M. Vrakopoulou, K. Margellos, J. Lygeros, G. Andersson, A probabilistic framework for reserve scheduling and security assessment of systems with high wind power penetration, Power Systems, IEEE Transactions on 28 (4) (2013) 3885-3896.

[23] A. J. Lamadrid, D. L. Shawhan, C. E. Murillo-Sánchez, R. D. Zimmerman, Y. Zhu, D. J. Tylavsky, A. G. Kindle, Z. Dar, Stochastically optimized, carbon-reducing dispatch of storage, generation, and loads, Power Systems, IEEE Transactions on 30 (2) (2015) 1064-1075.

[24] R. D. Zimmerman, C. E. Murillo-Sánchez, R. J. Thomas, MATPOWER: Steady-state operations, planning, and analysis tools for power systems research and education, Power Systems, IEEE Transactions on 26 (1) (2011) 12-19.

[25] R. Ferrero, S. Shahidehpour, V. Ramesh, Transaction analysis in deregulated power systems using game theory, Power Systems, IEEE Transactions on 12 (3) (1997) 1340-1347.

[26] V. Mohan, J. G. Singh, W. Ongsakul, An efficient two stage stochastic optimal energy and reserve management in a microgrid, Applied Energy 160 (2015) 28-38.

[27] G. Osório, E. Rodrigues, J. Lujano-Rojas, J. Matias, J. Catalão, New control strategy for the weekly scheduling of insular power systems with a battery energy storage system, Applied Energy 154 (2015) 459-470.

[28] M. Arbabzadeh, J. X. Johnson, R. De Kleine, G. A. Keoleian, Vanadium redox flow batteries to reach greenhouse gas emissions targets in an off-grid configuration, Applied Energy 146 (2015) 397-408.

[29] L. Johnston, F. Díaz-González, O. Gomis-Bellmunt, C. Corchero-García, M. Cruz-Zambrano, Methodology for the economic optimisation of energy storage systems for frequency support in wind power plants, Applied Energy 137 (2015) 660-669. 
[30] D. Wang, S. Parkinson, W. Miao, H. Jia, C. Crawford, N. Djilali, Hierarchical market integration of responsive loads as spinning reserve, Applied Energy 104 (2013) 229-238.

[31] Z. Hu, W. T. Jewell, Optimal power flow analysis of energy storage for congestion relief, emissions reduction, and cost savings, in: Power Systems Conference and Exposition (PSCE), 2011 IEEE/PES, IEEE, 2011, pp. 1-8.

[32] M. Black, G. Strbac, Value of bulk energy storage for managing wind power fluctuations, Energy Conversion, IEEE Transactions on 22 (1) (2007) 197-205.

[33] J. Cho, A. N. Kleit, Energy storage systems in energy and ancillary markets: A backwards induction approach, Applied Energy 147 (2015) 176-183.

[34] R. Walawalkar, J. Apt, R. Mancini, Economics of electric energy storage for energy arbitrage and regulation in New York, Energy Policy 35 (4) (2007) 2558-2568.

[35] J. Littlefield, R. Bhander, B. Bennett, T. Davis, L. Draucker, R. Eckard, W. Ellis, J. Kauffman, A. Malone, R. Munson, Life cycle analysis: existing pulverized coal (EXPC) power plant, National Energy Technology Laboratory.

[36] T. J. Skone, Role of alternative energy sources: Natural gas technology assessment, National Energy Technology Laboratory.

[37] PJM, PJM manual 12: Balancing operations, rev. 27 (December 2012).

[38] H. Banakar, N. Alguacil, F. D. Galiana, Electrothermal coordination part i: Theory and implementation schemes, Power Systems, IEEE Transactions on 20 (2) (2005) 798-805.

[39] J. B. Klein, The use of heat rates in production cost modeling and market modeling, Electricity Analysis Office, California Energy Commission (1998).

[40] Energy Information Administration, Electric power annual, http://www.eia.gov/ electricity/annual/ (March 2015). 
[41] M. Hummon, P. Denholm, J. Jorgenson, D. Palchak, B. Kirby, O. Ma, Fundamental drivers of the cost and price of operating reserves, Tech. Rep. NREL/TP-6A20-58491, NREL (2013).

[42] B. Dunn, H. Kamath, J.-M. Tarascon, Electrical energy storage for the grid: a battery of choices, Science 334 (6058) (2011) 928-935.

[43] PJM, PJM regulation data, http://www.pjm.com/markets-and-operations/ancillaryservices.aspx (2014).

[44] A. Papavasiliou, S. S. Oren, R. P. O’Neill, Reserve requirements for wind power integration: A scenario-based stochastic programming framework, Power Systems, IEEE Transactions on 26 (4) (2011) 2197-2206.

[45] M. Fripp, Greenhouse gas emissions from operating reserves used to backup large-scale wind power, Environmental Science \& Technology 45 (21) (2011) 9405-9412.

[46] C. Hitaj, Location matters: The impact of renewable power on transmission congestion and emissions, Energy Policy 86 (2015) 1-16.

[47] J. L. Mathieu, P. N. Price, S. Kiliccote, M. A. Piette, Quantifying changes in building electricity use, with application to demand response, Smart Grid, IEEE Transactions on 2 (3) (2011) 507518. 